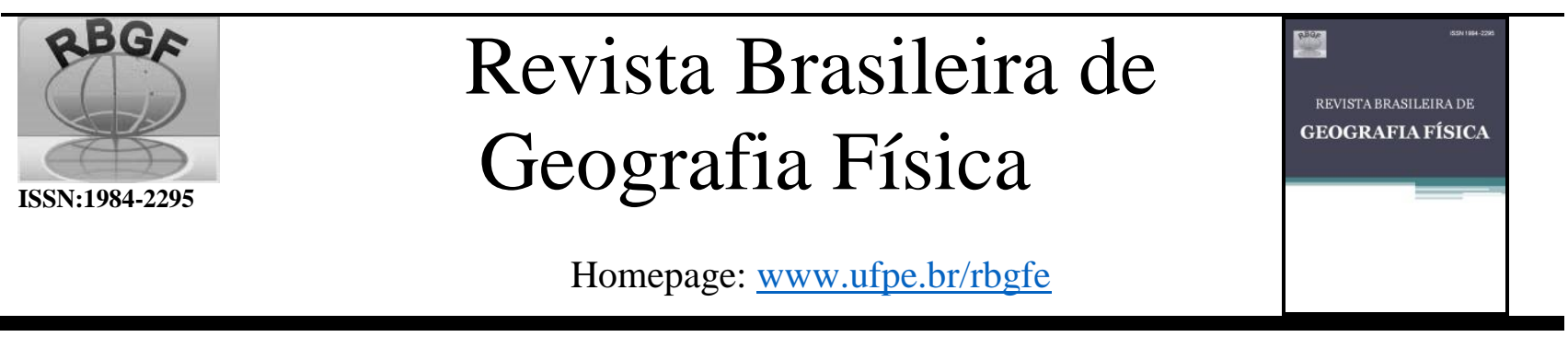

\title{
Precipitação Pluviométrica em Pelotas/RS: tendência, sistemas sinóticos associados e influência da ODP
}

Cassia Brocca Caballero, Mestranda do Programa de Pós-Graduação em Recursos Hídricos, Universidade Federal de Pelotas - UFPel, cassiabrocca@ hotmail.com;

Jéssica Fernanda Ogassawara, Mestranda do Programa de Pós-Graduação em Recursos Hídricos, Universidade Federal de Pelotas - UFPel, ogassawarajessica@gmail.com;

Viviane Rodrigues Dorneles, Doutoranda do Programa de Pós-Graduação em Recursos Hídricos, Universidade Federal de Pelotas - UFPel, vivianerdorneles@gmail.com;

André Becker Nunes, Professor do Programa de Pós-Graduação em Recursos Hídricos, Universidade Federal de Pelotas - UFPel, beckernunes@gmail.com

Artigo recebido em 09/11/2017 e aceito em 24/07/2018

\section{R E S U M O}

O estudo das alterações climáticas e de eventos extremos torna-se importante devido ao impacto que estes podem ocasionar. A partir da série de precipitação diária (1982 a 2015) de Pelotas/RS/Brasil, objetivou-se neste trabalho: (i) detectar as possíveis tendências na precipitação; (ii) relacionar os eventos extremos de precipitação aos sistemas meteorológicos que os ocasionaram; e (iii) analisar a influência da Oscilação Decadal do Pacífico (ODP) na variabilidade interanual. Para a análise de tendência, foi aplicada às séries total anual e trimestral o teste da regressão linear e de Mann-Kendall. Para análise dos eventos extremos foram selecionados os eventos de maior taxa de precipitação relacionando-os aos sistemas meteorológicos que os influenciaram mediante a análise dos campos de pressão ao nível médio do mar obtidos de dados de reanálise. A relação entre os valores anuais de ODP e os totais anuais de precipitação foi analisada de acordo com o coeficiente de Pearson. Os resultados mostraram que os dados analisados de precipitação total anual não possuem tendência significativa, porém, quando realizado os testes por trimestre, somente as precipitações do $3^{\circ}$ trimestre apresentaram tendência não significativa. Selecionou-se 18 eventos extremos ocorridos no período analisado, e desses, 12 apresentaram um padrão bem definido de centro de baixa pressão continental terminando como ciclone extratropical na costa do RS, e 9 ocorreram em anos de influência do El Niño. Além disso, os resultados obtidos indicaram uma relação significativa entre o índice ODP e a variabilidade da precipitação no munícipio de Pelotas.

Palavras-chave: chuvas intensas, El Niño Oscilação Sul, mudança climática, temperatura da superfície do mar, variabilidade da precipitação.

\section{The rainfall in Pelotas/RS: trend, associated synoptic systems and PDO influence}

\begin{abstract}
A B S T R A C T:
The study of climate change and extreme events is important due to the impacts related to them. Analyzing the daily precipitation series (1982 to 2015) of Pelotas/RS/Brazil, this work aims to: (i) detect possible trends in precipitation; (ii) relate the precipitation's extreme events to meteorological systems that caused them; and (iii) analyze the influence of Pacific Decadal Oscillation (PDO) on interannual precipitation variability. For trend analysis, the linear and MannKendall regression tests were applied to the total annual and quarterly precipitation series. For the analysis of extreme events, it was selected the events with highest precipitation rate, relating them to associated meteorological systems by analyzing the mean sea level pressure fields obtained from reanalysis dataset. The relationship between the annual PDO values and the total annual precipitation was analyzed using Pearson's coefficient. Results showed that the total annual precipitation do not have a significant trend, but when the tests were carried out by quarter, only the precipitations of the third quarter presented a non-significant trend. From the 18 selected extreme events occurred during the analyzed period, 12 presented a well defined pattern of continental low pressure center ending as extratropical cyclone on the
\end{abstract}


coast of RS, and 9 occurred in years of influence of El Niño. In addition, the results obtained indicated a significant relationship between the PDO index and the rainfall variability in the Pelotas.

Keywords: heavy rainfall, El Niño Southern Oscillation, climate change, sea surface temperature, precipitation variability.

\section{Introdução}

O clima é o principal determinante das atividades socioeconômicas da população, que se molda e adapta aos diferentes padrões mensais e sazonais de determinada região (Marengo et al. 2009). Nesse contexto, uma das variáveis mais consideráveis do clima é a precipitação, extremamente variável no tempo e no espaço e altamente influenciada por grandes massas de ar e fenômenos climáticos (Silva, 2013).

Atualmente, pesquisadores têm se dedicado aos estudos das alterações climáticas e suas consequências para a humanidade. Pode-se citar, como exemplo, eventos extremos (enchentes, secas, tufões, entre outros), que têm se mostrado cada vez mais frequentes, conforme a divulgação dos relatórios do Painel Intergovernamental sobre Mudanças Climáticas IPCC (IPCC, 2007).

Estas alterações climáticas são resultado tanto da atividade antrópica, principalmente devido ao aumento na emissão de Gases de Efeito Estufa (GEE) na atmosfera, quanto pela variabilidade climática natural do planeta.

Segundo Karl et al. (1996) e Houghton et al. (1996), o aquecimento global influencia na variação da distribuição da precipitação na superfície do planeta, podendo aumentar ou diminuir a ocorrência de chuva em determinadas regiões, acarretando no aumento dos eventos de secas e de cheias mais severas e prolongadas.

$\mathrm{Na}$ Região Sul do Brasil, impactos causados pela ocorrência de eventos extremos de precipitação - ou escassez - têm sido mais frequentes (Carvalho et al., 2002). Pode-se citar, como exemplo, as chuvas severas ocorridas em novembro de 2008 no Estado de Santa Catarina, além das secas vivenciadas em toda a região Sul no ano de 2012. Desse modo, destaca-se que as variações nos padrões regionais de precipitação interferem em diferentes setores de atividade humana, tais como na agricultura, abastecimento de água e luz.

A análise dos casos de eventos extremos de precipitação e o conhecimento dos processos que os ocasionaram, principalmente para áreas urbanas, é fundamental para o planejamento das condições de risco e identificação dos impactos sobre a drenagem da área. Este fato se justifica devido a probabilidade de eventos extremos ocasionarem severas inundações, representando uma ameaça para vida humana e infraestruturas (Da Silva e Nunes, 2011).

Segundo Dayan et al. (2015) a intensidade dos eventos extremos de precipitação em uma determinada região decorre, além das características do meio, da localização e profundidade dos sistemas meteorológicos. Além disso, da mesma forma, dependem das condições atmosféricas do local, em escala espacial e temporal, que induzem a precipitação. Esses aspectos desempenham um papel importante na formação do resultado de tais eventos de chuva extrema.

A análise de tendência em séries históricas de precipitação é uma das técnicas empregadas para se estimar possíveis ocorrências de mudança climática locais (Santos et al., 2009; Silva, R., 2013). A tendência climática é uma mudança caracterizada por um suave acréscimo ou decréscimo nos valores médios no período de registro (Back, 2011).

Dessa maneira, testes não paramétricos são os mais empregados pelos pesquisadores para avaliar a tendência das precipitações, como por exemplo, o teste de Mann-Kendall (Yue et al., 2002; Penereiro e Ferreira, 2012). Outro método também bastante aplicado para a avaliação de tendência é realizado através de análise de regressão (Penereiro e Ferreira, 2012).

A região sul do Brasil apresenta alta frequência de chuvas intensas (Cera e Ferraz, 2015). Seu regime pluviométrico é afetado por vários fenômenos atmosféricos, como, por exemplo, as Zonas de Convergência do Atlântico Sul (ZCAS) (Cataldi et al., 2010) e o El Niño Oscilação Sul (ENOS) (Grimm et al., 2000), influenciando assim a presença de outros sistemas meteorológicos (Berlato e Fontana, 2003), indicando uma alta variabilidade climática com relação à precipitação no Estado.

Teixeira (2004) observou que os principais sistemas responsáveis pelo volume de chuvas durante o ano na região sul do Brasil são os Sistemas Convectivos de Mesoescala (SCMs), caracterizados por intensos fluxos verticais 
turbulentos de calor e frentes frias e os Vórtices Ciclônicos de Altos Níveis (VCANs) que são responsáveis por precipitações de maior intensidade na região, porém com menor frequência.

Porém, além da forte influência desses fenômenos meteorológicos, na variabilidade da precipitação no Estado, no final da década de 1990 foi identificado um outro padrão recorrente de variabilidade climática interdecadal no Pacífico tropical, generalizado e detectável em uma variedade de sistemas climáticos e ecológicos. Esse padrão climático é denominado Oscilação Decadal do Pacífico - ODP (Mantua et al., 1997; Zang e Delworth, 2016).

O fenômeno ODP é caracterizado por duas fases, a positiva e a negativa. A fase positiva é caracterizada por anomalias negativas da temperatura da superfície do mar (TSM) no Pacífico Norte ocidental e central, cercadas por anomalias positivas da TSM ao longo da costa norte-americana, sendo a fase negativa o oposto (Zang e Delworth, 2016).

Os dados instrumentais da TSM no Pacífico Norte indicam que as fases alternadas da ODP podem durar de duas a três décadas com inversões de fase centradas em 1924/1925, 1946/1947, 1976/1977 e 1998/1999 (Linsley et al., 2015). Durante o século 20, foram identificadas uma fase fria (1947-1976) que separou duas fases quentes (1925-1946 e 19771998) da ODP (Mantua e Hare, 2002). De acordo com Molion (2005), a ODP aparenta ter novamente na fase fria a partir de 1999 , e deve perdurar até aproximadamente 2025.

Corroborando ao exposto, para série de 1982 a 2015, para o município de Pelotas/RS, o presente trabalho teve os seguintes objetivos: (i) detectar as possíveis tendências climáticas anuais na série de precipitação pluviométrica, empregando a análise de regressão e o teste nãoparamétrico de Mann-Kendall; (ii) analisar os tipos de sistemas meteorológicos que provocaram os eventos de precipitação de maior taxa observada em 48 horas; e (iii) analisar a influência da Oscilação Decadal do Pacífico na variabilidade interanual da precipitação no município.

\section{Material e métodos}

A cidade de Pelotas está localizada no estado do Rio Grande do Sul, Brasil, ocupando uma área total de $1.610,084 \mathrm{~km}^{2}$, com topografia predominantemente plana e de altitudes baixas. A altitude média é de $7 \mathrm{~m}$ acima do nível do mar e a cidade está localizada a cerca de $60 \mathrm{~km}$ do Oceano Atlântico. O clima da cidade é do tipo subtropical (Cfa), com clima temperado, onde a temperatura média do mês mais frio situa-se entre $18^{\circ} \mathrm{C} \mathrm{e}-3$ ${ }^{\circ} \mathrm{C}$, não há mês com escassez de precipitação (Gruppelli et al., 2003).

De acordo a classificação climática de Köppen (Kuintcher e Buriol 2001), o clima da região é subtropical (ou quase temperado), úmido, sem período seco. A precipitação média anual é de $1366,9 \mathrm{~mm}$, com a maior taxa de precipitação no mês de fevereiro e menor em outubro, 188,2 e $74,7 \mathrm{~mm}$, respectivamente. As temperaturas médias mensais são entre $10{ }^{\circ} \mathrm{C}$ e $22^{\circ} \mathrm{C}$ (Teixeira, 2011).

Os dados de precipitação foram adquiridos da Estação Agroclimatológica Convênio EMBRAPA/UFPel, INMET (31 ${ }^{\circ} 51^{\prime} \mathrm{S} ; 52^{\circ} 21^{\prime} \mathrm{O} ;$ a $13,2 \mathrm{~m}$ de altitude). Utilizaram-se dados de chuva do período de 1982 a 2015 da localidade de Pelotas/RS para a constituição da série de chuvas diárias e totais anuais.

\section{Análise de Tendência}

Para analisar as possíveis tendências nos dados de precipitação, foram considerados os totais anuais e totais por trimestre do ano. Em cada conjunto de dados em estudo, foram aplicadas a análise de regressão e realizado o teste de Mann-Kendall.

Conforme Back (2001), a análise de regressão é bastante aplicada para indicar as alterações climáticas por meio do teste de significância do coeficiente angular (a), sendo considerado a equação da reta. $\mathrm{O}$ teste em questão consiste em estabelecer o intervalo de confiança do coeficiente angular, sendo que a tendência dos dados só é significativa quando o intervalo do coeficiente apresentar valores maiores que zero.

O segundo teste aplicado foi o de MannKendall, o qual foi proposto inicialmente por Sneyers (1975). O teste de Mann-Kendall considera que, na hipótese de estabilidade de uma série temporal, a sucessão de valores ocorre de forma independente, e a distribuição de probabilidade deve permanecer sempre a mesma (série aleatória simples).

Sendo assim, os dados da série histórica em estudo são ordenados a partir do primeiro ano 
de observação até o último ano. $\mathrm{O}$ valor de $\mathrm{Yi}$, para cada tempo ti, é comparado com todos os outros dados coletados no tempo tj $(\mathrm{j}>\mathrm{i})$ consecutivos (Folhes e Fisch, 2006; Salviano et al. 2016). Posteriormente, é realizado o cálculo da estatística $\mathrm{S}$ de Mann-Kendall levando em consideração os seguintes critérios:

$$
S=\sum_{i=1}^{n-1} \sum_{j=i+1}^{n} \operatorname{sinal}(Y j-Y i)
$$

sinal $(Y j-Y i)=\left\{\begin{array}{rc}+1 & \text { se }(Y j-Y i)>0 \\ 0 & \text { se }(Y j-Y i)=0 \\ -1 & \text { se }(Y j-Y i)<0\end{array}\right.$

Séries históricas com grande número de termos (n), sob a hipótese nula $\left(\mathrm{H}_{0}\right)$ de ausência de tendência verdadeira, acabam apresentando $S$ de distribuição normal com média (s) zero e variância (Back, 2001; Salviano et al. 2016) conforme:

$\operatorname{Var}(S)=\frac{n(n-1)(2 n+5)}{18}$

Para o teste de significância estatística de S para a hipótese nula, é utilizado um teste bilateral, a qual pode ser rejeitada para grandes valores da estatística z (Back, 2001; Folhes e Fisch, 2006; Salviano et al., 2016), conforme a análise abaixo:

$\mathrm{z}=\left\{\begin{array}{cc}\frac{(\mathrm{s}-1)}{\sqrt{\operatorname{Var}(\mathrm{S})}} & \text { se } \mathrm{s}>0 \\ 0 & \text { se } \mathrm{s}=0 \\ \frac{(\mathrm{s}+1)}{\sqrt{\operatorname{Var}(\mathrm{S})}} & \text { se } \mathrm{s}<0\end{array}\right.$

Após a obtenção da análise da estatística z, é realizada a decisão final de aceitar ou rejeitar $\mathrm{H}_{0}$, isto é, pode-se confirmar a hipótese de estabilidade dos dados ou rejeita-la a favor da hipótese de existência de tendência nos dados (Salviano et al., 2016). Vale ressaltar que o sinal da estatística $\mathrm{z}$ indica se a tendência é crescente $(\mathrm{z}$ $>0)$ ou decrescente $(\mathrm{z}<0)$.

Sistemas Meteorológicos Associados à Eventos de Precipitação Intensa
Para a seleção dos casos de precipitação intensa que ocorreram no período, a série foi analisada tendo como critério para seleção os eventos que atingissem ou superassem um valor acumulado de $120 \mathrm{~mm}$ em dois dias consecutivos. A separação em dois dias consecutivos se dá pelo fato de que, na maioria das vezes, os sistemas meteorológicos que atingem uma área têm início em um determinado dia e cessam no dia seguinte.

Após a seleção, com base nas datas (dia, mês e ano) que esses ocorreram, foi possível associá-los ao fenômeno ENOS e a intensidade que o fenômeno atingiu (fraco, moderado, forte e muito forte) e posteriormente relacioná-los aos sistemas meteorológicos que os influenciaram. Os anos de influência do ENOS foram obtidos de acordo com o índice ONI (Oceanic Niño Index) do National Oceanic and Atmospheric Administration (NOAA).

Para a análise do sistema sinótico associado aos eventos de chuva intensa, foram obtidos os campos de pressão ao nível médio do mar (pnmm) obtidas dos dados de reanálises 2 do National Center for Environmental Prediction (NCEP) (Kanamitsu et al., 2002). Os dados possuem resolução espacial de $2.5^{\circ}$ e temporal de 6h. As imagens de satélite obtidas da Divisão de Satélites e Sistemas Ambientais do Instituto Nacional de Pesquisas Espaciais (DSA/INPE) colaboraram na identificação dos sistemas.

\section{Oscilação Decadal do Pacífico}

Os valores mensais do índice da ODP foram obtidos do Joint Institute for the Study of the Atmosphere and Ocean (2017), correspondentes ao período de 1982 a 2015. Agruparam-se valores mensais do índice ODP, obtendo-se os valores anuais. Com os dados de precipitação, obteve-se a série de totais anuais a partir da soma dos totais diários para o período. Além disso, calculou-se média do período em análise.

Posteriormente, calculou-se o coeficiente de correlação linear (coeficiente de Pearson) para avaliar o grau de associação entre as variáveis. Este coeficiente varia de -1 a 1 e, quanto mais próximo de 1, maior é a correlação positiva entre as variáveis analisadas. Aplicou-se também o teste $t$ de Student para testar a significância da correlação linear. 
Entretanto, Xavier e Xavier (2004) reconhecem que a existência de correlação não implica, necessariamente, em efeito causal, pois existe um "lag" entre o intervalo utilizado para o índice médio da ODP e os intervalos utilizados para a TSM. Dessa forma, avaliou-se também a correlação entre a precipitação total anual e o índice ODP com um "lag" de dez anos. Este valor foi obtido por Streck et al. (2009) para a cidade de Santa Maria, no Rio Grande do Sul. Optou-se por utilizar o mesmo lag devido à distância relativamente pequena entre as cidades.

\section{Resultados e discussão}

\section{Análise de Tendência}

A Tabela 1 apresenta os resultados referentes ao teste de Mann-Kendall e a análise de regressão linear, em relação a precipitação anual e também aos trimestres dos anos em estudo. E na Figura 1 são mostrados os gráficos dos resultados do teste de Mann-Kendall e da análise de regressão.

Diante da análise dos resultados, é possível perceber que houve tendência significativa e crescente para a análise dos dados da precipitação total anual e para a análise da precipitação total do $3^{\circ}$ e $4^{\circ}$ trimestre, pois os intervalos dos coeficientes (Figura 1a) e o valor de $\mathrm{z}$ adquirido pelo teste de Mann-Kendall apresentaram valores maiores que zero. Porém, observando os gráficos de regressão linear (Figura $1 \mathrm{~b}$ e 1e) nota-se que a tendência nos dados, tanto da análise da precipitação total anual quanto da análise do $3^{\circ}$ trimestre, não é significativa, visto que a reta possui pouca inclinação, ou seja, os valores do coeficiente angular são baixos. As análises do $1^{\circ}$ e $2^{\circ}$ trimestre indicaram tendência negativa e decrescente (Figura 1c e 1d), pois os valores adquiridos de $\mathrm{z}$ e dos coeficientes foram negativos. Já os resultados obtidos de $\mathrm{z}$ e do coeficiente do $4^{\circ}$ trimestre indicaram que a série analisada possui uma tendência positiva e crescente (Figura 1f).

Conforme estudos de Berlato et al. (1995), onde foi analisado dados de precipitação de 17 locais do Rio Grande do Sul, não foi detectado tendência de longo prazo na precipitação total anual, sendo que as reduções e incrementos que foram identificados em curto período, provavelmente se referem à flutuação da precipitação pluvial. Todos estas pesquisas e análise de dados mostram que grande maioria das alterações têm caráter regional, ou seja, não estão ligadas a uma mudança global do clima (Back, 2001).

Tabela 1. Resultados do teste de Mann-Kendall e da análise de regressão

\begin{tabular}{lcccc}
\hline \multirow{2}{*}{ Variável analisada } & \multicolumn{2}{c}{ Teste não-paramétrico } & \multicolumn{2}{c}{ Análise de regressão } \\
\cline { 2 - 5 } & $\begin{array}{c}\text { Mann-Kendall } \\
\text { (z calculado) }\end{array}$ & Tendência & $\begin{array}{c}\text { Coeficiente } \\
\text { angular }\end{array}$ & $\begin{array}{c}\text { Intervalo de } \\
\text { confiança (95\%) }\end{array}$ \\
\hline Precipitação total anual & 0,148 & $\mathrm{NS}^{(1)}$ & 1,6061 & $-0,312$ a 0,408 \\
Precipitação total $1^{\circ}$ trimestre & $-0,059$ & $-\mathrm{S}^{(2)}$ & $-0,6621$ & $-0,406$ a 0,314 \\
Precipitação total $2^{\circ}$ trimestre & $-0,059$ & $-\mathrm{S}$ & $-0,3635$ & $-0,385$ a 0,335 \\
Precipitação total $3^{\circ}$ trimestre & 0,430 & $\mathrm{NS}$ & 0,0863 & $-0,354$ a 0,366 \\
Precipitação total 4 trimestre & 0,563 & $+\mathrm{S}^{(3)}$ & 2,1244 & $-0,213$ a 0,500 \\
\hline (1) Tendência não significativa & & & & \\
(2) Tendência negativa & & & & \\
(3) Tendência positiva & &
\end{tabular}




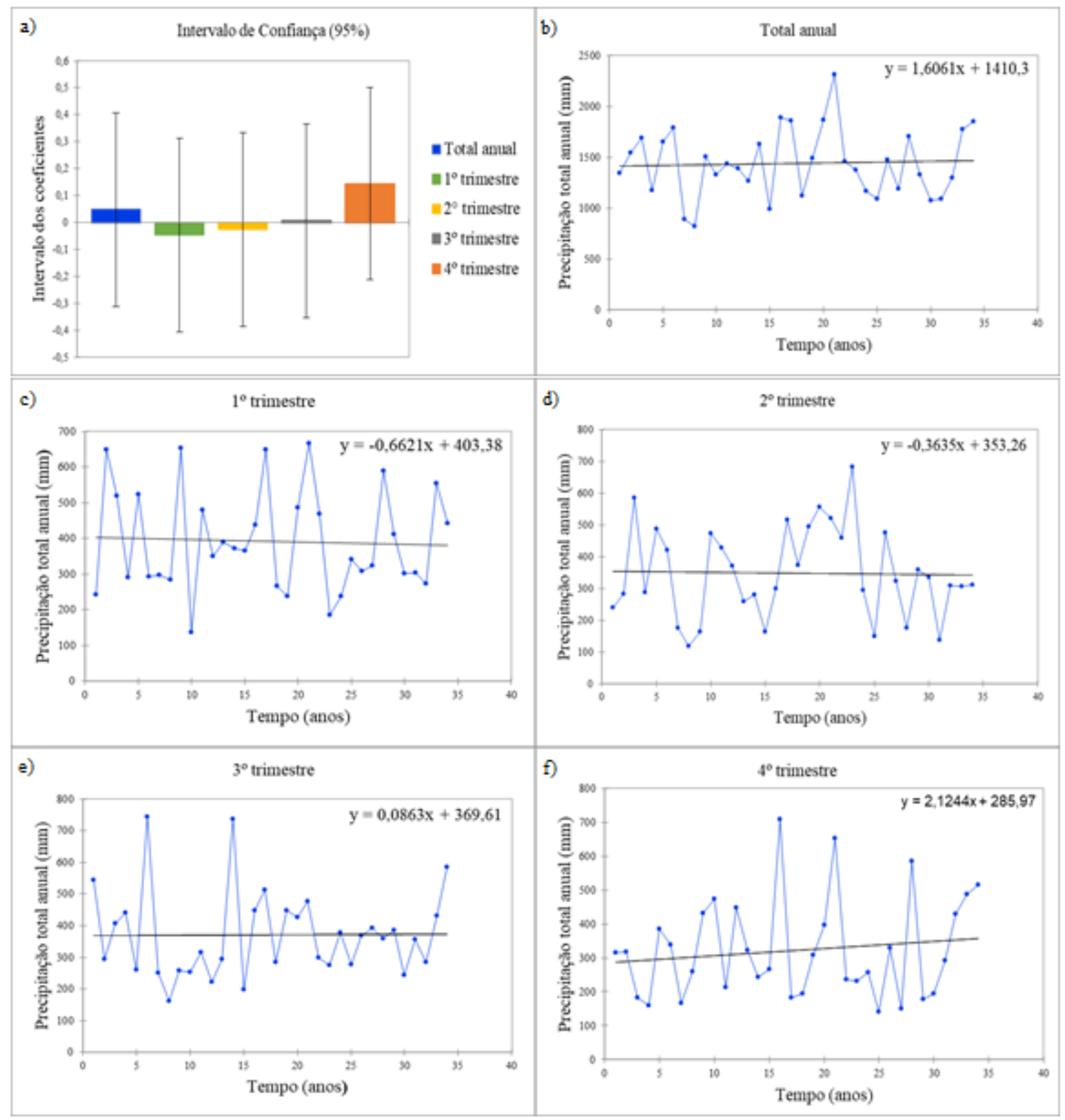

Figura 1: Gráficos dos resultados do teste de Mann-Kendall e da análise de regressão

Sistemas Meteorológicos Associados à Eventos de Precipitação Intensa

Foram selecionados 18 casos de eventos de precipitação intensa $(120 \mathrm{~mm}$ acumulados em $48 \mathrm{~h}$ ) que atingiram a localidade em estudo durante o período analisado. A Tabela 2 apresenta os eventos extremos selecionados, suas respectivas datas, o valor precipitado, relação aos anos de influência de ENOS, os sistemas associados aos eventos de precipitação e o tipo de nebulosidade.

Na Tabela 2 pode-se observar que o mês que apresentou maior número de casos de eventos extremos foi o mês de fevereiro, com quatro registros (1983 com dois eventos, 1997 e 2003), tendo o verão como a estação do ano com maior probabilidade de eventos extremos neste período. Nunes e Da Silva (2013) estudaram o Estado de Catarina e verificaram que o verão também foi a 
estação que mais apresentou casos de eventos extremos de precipitação. Por outro lado, Teixeira (2004) analisou a distribuição sazonal de eventos intensos de chuva na Região Sul do Brasil, e essas apresentaram maior número nas estações primavera e outono.

Sobre a comparação dos eventos extremos com a influência do fenômeno ENOS e a intensidade que o esse atingiu (Tabela 2), pode-se observar que nove eventos extremos, metade do total, estão associados ao período de influência do El Niño. Sendo do ano de 1997 El Niño muito forte, 1983, 1987 e 2015 El Niño forte e moderado, 1998, 2009 El Niño moderado e 2003 El Niño fraco. Os demais eventos estão associados a influência de anos neutros e um evento ocorreu em um ano de influência do La Niña.

Observa-se também que os dois casos de Frente Fria - Transição Baixa Térmica Ciclogênese Extratropical (1997 e 2015) ocorreram em anos de influência do El Niño. A nebulosidade associada a SCM ocorreram em dois anos Neutros (1997 e 2004) e duas ocorreram no ano de 2015, que também foi um ano de influência do El Niño.

Vários pesquisadores verificaram a influência do fenômeno ENOS na precipitação na Região Sul do Brasil (Grimm et al., 1998; Teixeira, 2004; Lopes, 2006; Fischer et al., 2008; Da Silva, 2011), sendo observada forte relação entre a ocorrência de eventos intensos de precipitação com anos de El Niño, bem como quando houve uma diminuição do número de eventos o fenômeno associado foi o La Niña. Nesta análise foram encontrados como sistemas meteorológicos, as baixas térmicas, frentes frias, ciclone subtropical e extratropical e o processo de ciclogênese.

Diante dos resultados da Tabela 2, observa-se que, destes 18 casos de eventos extremos de precipitação, a maioria dos eventos (12 casos) apresentaram um padrão bem definido de centro de baixa pressão: iniciaram-se como Baixa do Chaco (Baixa Térmica, BT) e terminaram como ciclone extratropical na costa do RS. Em outras palavras, observou-se que a Baixa do Chaco começou a se prolongar na direção sudeste, passando pelo RS e vindo a tornar-se um ciclone extratropical (sistema bem circular, simétrico, diferentemente da Baixa do Chaco) na costa do RS. Esses casos foram chamados de Transição Baixa Térmica-Ciclone Extratropical.

Para análise visual das imagens de satélite desses 12 casos de transições, foram geradas imagens de 6 em 6 horas, a partir da 00Z (hora de Greenwich), 06Z, 12Z e 18Z dos dois dias de cada caso (independente da data de ocorrência), mais o $00 \mathrm{Z}$ do terceiro dia, que representa as $21 \mathrm{~h}$ (hora local) do dia 2. Destas imagens foram gerados campos compostos (Figura 2), que são campos médios de cada passo de tempo.

A Figura 2 mostra os campos compostos de pnmm, onde pode-se observar que o evento culmina com um CE na costa do RS.

Dos casos de precipitação intensa encontrados, nenhuma nebulosidade foi associada a CCM (Complexo Convectivo de Mesoescala) (Tabela 2), porém foram observados alguns casos de Sistemas Convectivos de Mesoescala genéricos (1997, 2004 e 2015), e em outros casos se tratou de uma nebulosidade genérica associada a um sistema frontal.

\section{Oscilação Decadal do Pacífico}

A Figura 3 apresenta os totais anuais de precipitação em Pelotas e o índice ODP médio de cada ano na série histórica, desde 1982 até 2015.

Observa-se a alta variabilidade interanual da precipitação ao longo da série histórica, apresentando a mínima precipitação no ano de 1989, com um total anual de $823 \mathrm{~mm}$, e a máxima em 2002, totalizando $2315,5 \mathrm{~mm}$ naquele ano. A partir da análise do gráfico, pode-se verificar visualmente uma relação direta entre as fases positivas e negativas da ODP com a variabilidade interanual da precipitação. $O$ coeficiente de Pearson calculado foi de 0,305 , mostrando que as variáveis têm uma correlação positiva entre si. Esta correlação foi significante de acordo teste $t$ de Student para um nível de significância de $95 \%$. Utilizando o "lag" de 10 anos, sugerido por Streck et al. (2009), obteve-se um coeficiente de correlação de $-0,225$. Este resultado sugere que a precipitação e o índice ODP possuem uma correlação negativa, porém futuras analises devem 
Tabela 2. Eventos extremos de precipitação selecionados na análise da série de precipitação, para o período de 1982 a 2015, suas datas de ocorrência, valor precipitado (mm), influência do ENOS, sistemas meteorológicos responsáveis e nebulosidade, para o município

\begin{tabular}{ccccccccc}
\hline Caso & ANO & DIA & MÊS & P $(\mathrm{mm})$ & ENOS & Intensidade & Sistema & Nebulosidade \\
\hline 1 & 1983 & $14 / 15$ & Fev & 242,8 & El niño & Forte & TBT-CS & sem dados \\
2 & 1983 & $23 / 24$ & Fev & 153,2 & El niño & Forte & BT & sem dados \\
3 & 1986 & $16 / 17$ & Mai & 123,4 & Neutro & - & TBT-CE & sem dados \\
4 & 1987 & $28 / 29$ & Ago & 131,6 & El niño & Forte & TBT-CE & sem dados \\
5 & 1993 & $01 / 02$ & Jan & 129,4 & Neutro & - & BT & sem dados \\
6 & 1995 & $14 / 15$ & Jul & 159,2 & Neutro & - & TBT-CE & sem dados \\
7 & 1997 & $13 / 14$ & Fev & 120,6 & Neutro & - & TBT-CE & SCM \\
8 & 1997 & $21 / 22$ & Dez & 154,6 & El niño & Muito Forte & FF-TBT-CE & Genérica \\
9 & 1998 & $16 / 17$ & Abri & 154,5 & El niño & Moderado & TBT-CE & Genérica \\
10 & 1998 & $25 / 26$ & Ago & 123,0 & La niña & Moderado & TBT-CE & Genérica \\
11 & 2003 & $05 / 06$ & Fev & 121,6 & El niño & Fraco & BT & Genérica \\
12 & 2004 & $06 / 07$ & Mai & 257,0 & Neutro & - & CS & SCM \\
13 & 2005 & $10 / 11$ & Set & 123,8 & Neutro & - & TBT-CE & Genérica \\
14 & 2007 & $18 / 19$ & Mar & 133,8 & Neutro & - & CS & Genérica \\
15 & 2009 & $29 / 30$ & Jan & 170,0 & Neutro & - & TBT-CE & Genérica \\
16 & 2009 & $04 / 05$ & Nov & 153,4 & El niño & Moderado & BT & SCM e LI \\
17 & 2015 & $19 / 20$ & Jul & 124,7 & El niño & Moderado & FF-TBT-CE & Genérica \\
18 & 2015 & $23 / 24$ & Set & 133,7 & El niño & Forte & TBT-CE & 2 SCM's \\
\hline
\end{tabular}

TBT-CS: Transição Baixa Térmica-Ciclone Subtropical; BT: Baixa Térmica; TBT-CE: Transição Baixa Térmica-Ciclone Extratropical; FF-TBT-CE: Frente Fria-Transição Baixa Térmica-Ciclogênese Extratropical; CS: Ciclone Subtropical; SCM: Sistema Convectivo de Mesoescala; LI: Linha de Instabilidade.

ser realizadas a fim de fornecer um melhor entendimento desse resultado,

Considerando que o índice ODP possui uma influência positiva na precipitação, constatam-se tendências entre a fase negativa e positiva da ODP com a diminuição e o aumento da precipitação, respectivamente. A fase negativa da ODP se estendeu de 1961 a 1976, em que se espera uma influência na diminuição da precipitação, no entanto esta fase não está compreendida no período analisado neste estudo.

Já a fase positiva se estende de 19771998, em que se verifica uma precipitação acima da normal durante esses anos. Porém, nota-se que em alguns anos essa relação não é observada, como em 1988, 1989, 1996 e 1999. Isso se dá pelo fato de que esses foram anos de La Niña, provocando anomalias negativas de precipitação, o que influencia na redução da precipitação na região sul do Brasil (Berlato e Fontana, 2003).

A partir de 1999, observa-se que a ODP entra novamente em sua fase negativa, apenas apresentando algumas anomalias positivas. Porém, observa-se nessa fase um número significativo de precipitações anuais acima da média. Como exemplo, o ano de 2002, ano em que houve a precipitação máxima do período analisado. Apesar de estar em uma fase fria da ODP, esse foi um ano de El Niño moderado (GGWeather, 2017), fenômeno que tende a causar um aumento da precipitação na região $\mathrm{Sul}$ do Brasil.

Dessa forma, pode-se observar que o regime de precipitação no munícipio de Pelotas, 
Revista Brasileira de Geografia Física v.11, n.04 (2018) 1429-1441.

além de ter influência direta do fenômeno ENOS, também apresenta correção com o índice ODP, que interfere significativamente na variação da precipitação durante os anos. O entendimento do efeito de fenômenos climáticos sobre o regime de precipitação é essencial para a compreensão de sua variabilidade e torna-se uma ferramenta importante para predição de possíveis anomalias, tanto negativas quanto positivas, que venham a interferir as atividades humanas e que, de alguma forma, causem algum tipo de prejuízo.
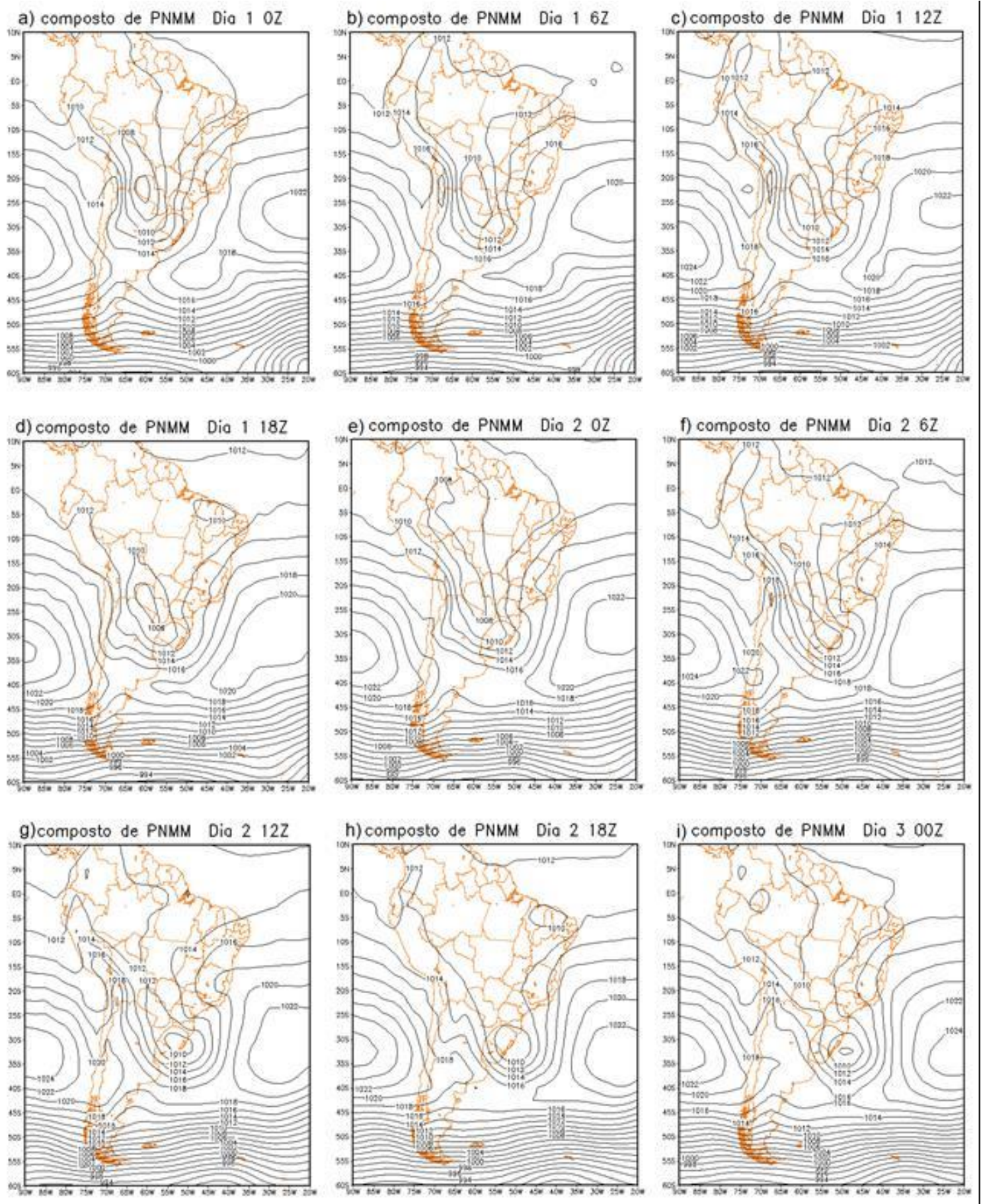

Figura 2. Campos compostos de pnmm para: a) dia 1 às 00Z; b) dia 1 às 06Z; c) dia 1 às 12Z; d) dia 1 às 18Z; e) dia 2 às 00Z; f) dia 2 às 06Z; g) dia 2 às 12Z; h) dia 2 às 18Z; i) dia 3 às $00 Z$. 


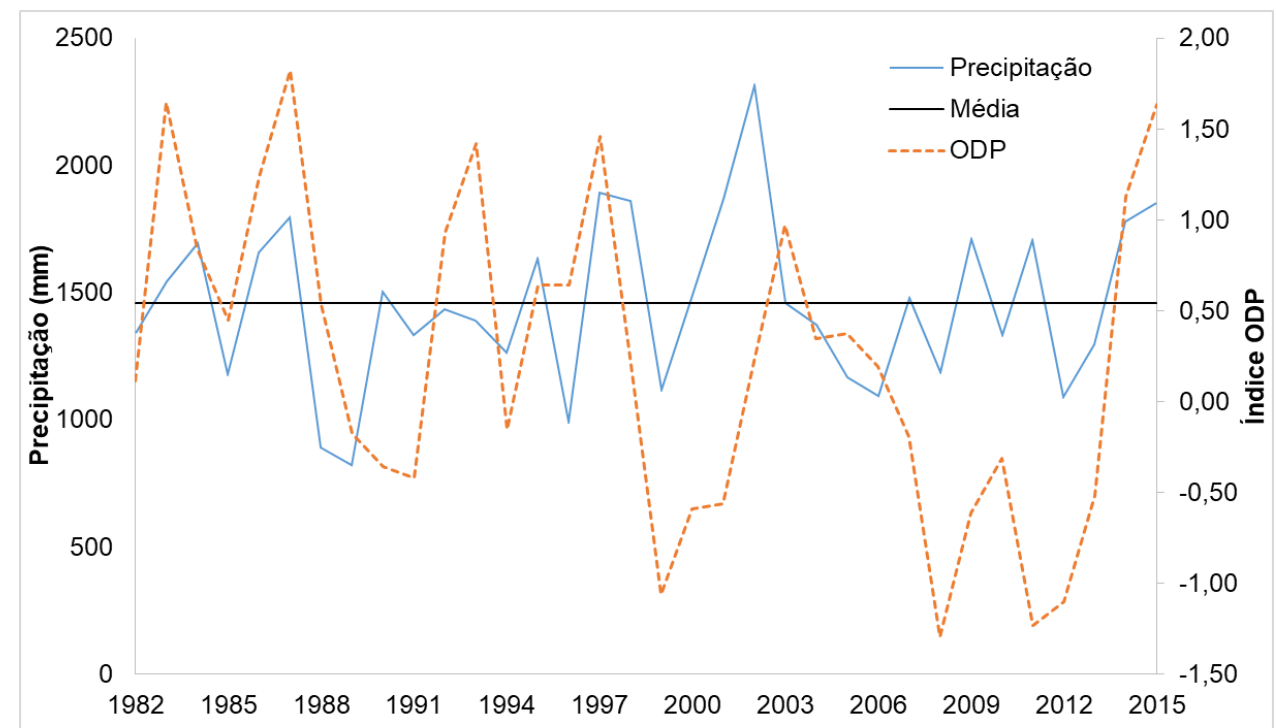

Figura 3. Total anual de precipitação em Pelotas, RS e ODP, índice Oscilação Decadal do Pacífico para a relação sem lag. A linha horizontal representa a média de chuvas do período de 1982-2015.

\section{Conclusão}

A partir dos resultados finais adquiridos, foi possível observar que os dados analisados da precipitação total anual não possuem tendência significativa, ou seja, os dados da série em análise são homogêneos o que implica que a mesma não possui extremos significantes. Porém, quando realizado os testes por trimestre, somente as precipitações do $3^{\circ}$ trimestre apresentaram tendência não significativa. Já as precipitações do $1^{\circ}$ e $2^{\circ}$ trimestre apresentaram tendência negativa e decrescente e as do $4^{\circ}$ trimestre apresentaram tendência positiva e crescente. Também foi possível observar que existe uma concordância entre os resultados obtidos, dessa maneira, ambos os métodos de análise de tendências mostraram-se apropriados para serem aplicados em estudos referentes a identificação de tendências de séries de precipitação.

Sobre a análise dos eventos meteorológicos, os resultados do presente trabalho mostraram que dos 18 casos de chuva maior que $120 \mathrm{~mm}$ em 48 horas em Pelotas-RS, no período de 1982-2015, 12 estiveram associados à transição Baixa Térmica - Ciclone Extratropical. Em que a maioria desses casos ocorreram sob condições de El Niño.

Além disso, os resultados obtidos indicaram uma relação significativa entre o índice ODP e a variabilidade da precipitação no munícipio de Pelotas. Observou-se que na fase negativa da ODP houve uma precipitação abaixo da média e, na fase positiva, acima. Em alguns momentos constatou-se que a precipitação não acompanhou a tendência do índice, o que é relacionado com a forte influência que o fenômeno ENOS tem sobre o Sul do País.

Apesar da obtenção de resultados satisfatórios, é importante ressaltar que estudos futuros devem ser realizados para o melhor entendimento da influência da ODP na variabilidade da precipitação, principalmente na análise do "lag" entre o índice médio da ODP e os intervalos utilizados para a TSM.

\section{Agradecimentos}

Os autores agradecem ao programa de Pós-Graduação em Recursos Hídricos da Universidade Federal de Pelotas (UFPel) pela oportunidade de aprendizado, desenvolvimento e pesquisa.

\section{Referências}

Back, A. J., 2001. Aplicação de análise estatística para identificação de tendências climáticas. Revista Pesquisa Agropecuária Brasileira [online] 36, 717-726. Disponível: http://dx.doi.org/10.1590/S0100- 
204X2001000500001. Acesso em: 8 nov. 2017.

Berlato, M. A., Fontana, D. C., Bono, L., 1995. Tendência temporal da precipitação pluvial anual no Estado do Rio Grande do Sul. Revista Brasileira de Agrometeorologia, Santa Maria 3, 111-113.

Berlato, M.A., Fontana, D.C., 2003. El Niño e La Niña: impactos no clima, na vegetação e na agricultura do Rio Grande do Sul; aplicações de previsões climáticas na agricultura. UFRGS, Porto Alegre.

Carvalho, L.M.V.; Jones, C.; Liebmann, B. 2002. Extreme precipitation events in Southeastern South America and large-scale convective patterns in the South Atlantic Convergence Zone. Journal of Climate, 15, 2377-2394. Disponível: https://doi.org/10.1175/15200442(2002)015<2377:EPEISS>2.0.CO;2.

Acesso em: 15 dez. 2017.

Cataldi, M., Assad, L. P. F., Torres Junior, A. R., Alves, J. L. D., 2010. Estudo da influência das anomalias da TSM do Atlântico Sul extratropical na região da Confluência BrasilMalvinas no regime hidrometeorológico de verão do Sul e Sudeste do Brasil. Revista Brasileira de Meteorologia [online] 25, 513524. ISSN 0102-7786. Disponível: http://dx.doi.org/10.1590/S0102-

77862010000400010 . Acesso em: 09 set. 2017.

Cera, J. C., Ferraz, S. E. T., 2015. Variações climáticas na precipitação no sul do Brasil no clima presente e futuro. Revista Brasileira de Meteorologia 30, 81-88. Disponível: http://dx.doi.org/10.1590/0102-778620130588. Acesso em: 09 set. 2017.

Da Silva, G. C., 2011. Análise quantitativa de eventos extremos de precipitação da região Leste e Norte de Santa Catarina e projeções climáticas de acordo com modelagem climática regional. Dissertação (Mestrado). Universidade Federal de Pelotas - UFPel.

Da Silva, G. C., Nunes A. B., 2011. Análise de eventos extremos de precipitação no leste de Santa Catarina: estudo de tendência; Ciência e Natura, esp, 251-254. Disponível:
http://dx.doi.org/10.5902/2179460X9432.

Acesso em: 17 out. 2017.

Dayan, U., Nissen, K.M., Ulbrich, U., 2015. Atmospheric conditions inducing extreme precipitation over the Eastern and Western Mediterranean. Natural Hazards and Earth System Sciences Discussions 3, 3687-3732. Disponível: https://doi.org/10.5194/nhess-152525-2015. Acesso em: 02 nov. 2017.

Fischer, G. R., Diniz, G. B., Marques, J. R. Q., 2008. Previsão do número de dias de chuva para a metade sul do Rio Grande do Sul utilizando a temperatura da superfície do mar. Revista Brasileira de Meteorologia [online] 23, 143-151. Disponível: http://dx.doi.org/10.1590/S0102-

77862008000200003. Acesso em: $15 \mathrm{dez}$. 2017.

Folhes, M. T., Fisch, G., 2006. Caracterização climática e estudo de tendências nas séries temporais de temperatura do ar e precipitação em Taubaté (SP). Revista Ambiente e Água, 1, 61-71.

GGWeather - Golden Gate Weather Services. El Niño and La Niña Years and Intensities Based on Oceanic Niño Index (ONI), 2017. Disponível em: http://ggweather.com/enso/oni.htm. Acesso em: 09 set. 2017.

Grimm, A. M., Barros, V. R., Doyle, E., 2000. Climate variability in southern South America associated with El Niño and La Niña events. Journal of Climate 13, 35-58. Disponível: https://doi.org/10.1175/15200442(2000)013<0035:CVISSA>2.0.CO;2. Acesso em: 02 nov. 2017.

Grimm, A., Ferraz, S. E. T., Gomes, J., 1998. Precipitation anomalies in Southern Brazil associated with El Niño and La Niña events. Journal of Climate, Boston 11, 2863-2880. Disponível: $\quad$ https://doi.org/10.1175/15200442(1998)011<2863:PAISBA>2.0.CO;2. Acesso em: 17 out. 2017.

Houghton, J. T., Meira Filho, L. C., Callander, B. A., Harris, N., Katternberg, A., Maskell, K., 1996. Climate change 1995: The science of 
climate change: contribution of working group I to the second assessment report of the Intergovernmental Panel on Climate Change. 2. ed. Cambridge University Press.

Intergovernmental Panel On Climate Change (IPCC), 2007. Summary for Policymakers, in Climate Change 2007: The Physical Science Basis, Contribution of Working Group I to the Fourth Assessment Report of the Intergovernmental Panel on Climate Change. Cambridge (UK), Cambridge University Press.

Kanamitsu, M. Ebisuzaki, W., Woollen, J., Yang, S., Hnilo, J. J., Fiorino, M., Potter, G. L., 2002. NCEP-DOE AMIP-II Reanalysis (R-2). Bulletin of the American Meteorological Society 83, 1631-1643. Disponível: https://doi.org/10.1175/BAMS-83-11-1631.

Acesso em: 02 nov. 2017.

Karl, T. R., Knigth, R. W., Easterling, D. R., Quayle, R. G., 1996. Indices of climate change for the United States. American Meteorological Society Bulletin [online] 77. Disponível: $\quad$ https://doi.org/10.1175/15200477(1996)077<0279:IOCCFT>2.0.CO;2.

Acesso em: 17 out. 2017.

Kuintcher, A., Buriol, G.A., 2001. Clima do estado do Rio Grande do Sul, segundo a classificação climática de Koppen e Thornthwaite. Disciplinarum Scientia, Série, Ciências exatas: Santa Maria, 2, 171-182.

Linsley, B.K., Wu, H.C., Dassié, E.P., Schrag, D.P., 2015. Decadal changes in South Pacific sea surface temperatures and the relationship to the Pacific decadal oscillation and upper ocean heat content. American Geophysical Union 42, 2358-2366. Disponível: https://doi.org/10.1002/2015GL063045. Acesso em: 02 nov. 2017.

Lopes, F. Z., 2006. Relações entre o Multivariate Enso Index (MEI) e a TSM das regiões dos Niños com a precipitação em regiões homogêneas do estado do Rio Grande do Sul. Dissertação (Mestrado). Pelotas. Universidade Federal de Pelotas - UFPel.

Mantua, N. J., Zhang, Y., Wallace, J. M., Francis, R. C. 1997. A Pacific interdecadal climate oscillation with impacts on salmon production. Bulletin of the American Meteorological Society 78, 1069-1079. Disponível: https://doi.org/10.1175/15200477(1997)078<1069:APICOW>2.0.CO;2. Acesso em: 09 set. 2017.

Mantua, N. J., Hare S. R., 2002. The Pacific Decadal Oscillation. Journal of Oceanography $58,35-44$.

Marengo, J. A.; Schaeffer, R.; Pinto, H. S.; Zee, D. M. W. Mudanças climáticas e eventos extremos no Brasil. Rio de Janeiro: FBDS, 2009. $76 \mathrm{p}$.

Molion, L. C. B., 2005. Aquecimento Global, El Niños, Manchas Solares, Vulcões e Oscilação Decadal do Pacífico. Revista Climanálise 3, 516.

Nunes A. B., Da Silva G. C., 2013. Climatologia dos eventos extremos de precipitação na região Leste e Norte de Santa Catarina: Clima Presente e Futuro. Revista Brasileira de Geofísica 31, 413-425. Disponível: http://dx.doi.org/10.1590/0102-778620130588. Acesso em: 09 set. 2017.

Penereiro, J. C., Ferreira, D. H. L., 2012. Estatística apoiada pela tecnologia: uma proposta para identificar tendências climáticas. Acta Scientiae 13, 87-105.

Salviano, M. F., Groppo, J. D., Pellegrino, G. Q., 2016. Análise de Tendências em Dados de Precipitação e Temperatura no Brasil. Revista Brasileira de Meteorologia [online] 31. Disponível: http://dx.doi.org/10.1590/0102778620150003. Acesso em: 23 nov. 2017.

Santos, C. A. C., Brito, J. I. B., Rao, T. V. R., Menezes, H. E. A., 2009. Tendências dos índices de precipitação no Estado do Ceará. Revista Brasileira de Meteorologia [online] 24. Disponível: https://doi.org/10.1590/S010277862009000100004. Acesso em: 01 out. 2017.

Silva, J. R., 2013. Análise climática pontual de eventos extremos de precipitação no Rio Grande do Sul. Dissertação (Mestrado). 
Pelotas. Faculdade de Meteorologia, Universidade Federal de Pelotas.

Silva, R. O. B., 2013. Tendências de mudanças climáticas na precipitação pluviométrica no estado de Pernambuco. Dissertação (Mestrado) Pernambuco. Universidade Federal de Pernambuco.

Sneyers, R., 1975. Sur analyse statistique des series observations. Genève: Organisation Météorologique Mondial. Monografia. Geneve, OMM.

Streck, N. A., Buriol, G. A., Heldwein, A. B., Gabriel, L. F., Paula, G. M., 2009. Associação da variabilidade da precipitação pluvial em Santa Maria com a Oscilação Decadal do Pacífico. Pesquisa Agropecuária Brasileira [online] 44, 1553-1561. Disponível: http://dx.doi.org/10.1590/S0100-

204X2009001200001. Acesso em: 09 set. 2017.
Teixeira, M. S. 2004. Atividade de ondas sinópticas relacionada a episódios de chuvas intensas na Região Sul do Brasil. Dissertação (Mestrado) São José dos Campos. Instituto Nacional de Pesquisas Espaciais - INPE.

Zhang, L. E., e Delworth, T. L., 2016. Response of the Pacific Decadal Oscillation to Climate Change. Journal of Climate 29, 5999-6017. Disponível: https://doi.org/10.1175/JCLI-D15-0690.1. Acesso em: 27 set. 2017.

Yue, S., Pilon, P., Cavadias, G., 2002. Power of the Mann-Kendall and Spearman's rho tests for detecting monotonic trends in hydrological series. Journal of Hydrology [online] 259. Disponível: https://doi.org/10.1016/S00221694(01)00594-7. Acesso em: 06 out. 2017. 